\title{
The Influence and Application of Plug-in Technology on Computer Software
}

\author{
XinLin Zhang \\ Zhumadian Vocational and Technical College He Nan 463000
}

Keywords: Plug-in technology; Computer software; Application; Influence

\begin{abstract}
Plug-in technology is an application that carries a lot of functionality. With the development of plug-in technology, it has a greater influence on computer software. This paper analyzes the concept and connotation of plug-in technology, and brings forward its great influence on the computer software based on its many functions, which in turn is the study of its application.

Plug-in technology is the application of modern computer software programs. Due to the application of plug-in technology, the related functions of the computer software has been greatly expanded, and it has more application. Based on the analysis of the connotation of computer software plug-in technology, this paper expatiates the principle and basic types of the plug-in technology, and then analyzes its application trend in real world and the focus of applied research.
\end{abstract}

\section{Computer Software Plug-in Technology}

A plug-in is a program that is written based on a program-specific application program interface. Plug-ins are very common in modern software, there are many types of plug-ins. The purpose of the plug-in is to develop and implement the functions that the original pure system platform does not have.

Computer software is a program in a computer system. Plug-ins are also very common access points in these systems. To a certain extent, the computer software plug-in can achieve connection software program through specific interface, so as to meet the expansion and upgrade of computer software system, the computer technology more practical and operational, to better meet the needs of the majority of computer users. In the process of computer software development, how to configure the plug-in with the corresponding function into the system is related to the speed and efficiency of the computer software operation and the function of the maintenance of the computer software.

With the development of plug-in technology, its use in computer software got great attention and popularity, because it can realize the function of computer software cannot be achieved. It can be said that now the computer software is essentially a plug-in. It can be said that there is no plug-in technology, a lot of computer software functions cannot be better demonstrated. Computer software is the core of the computer system operation, the plug-in technology will further the development of these functions and make the computer system functions, promote further optimization of computer system function, performance further improved.

\section{The Principle and Type of Computer Software Plug - in Technology}

How to play the role of computer software plug-in technology? How does it work? And what types are there? This is very helpful for us to deeply grasp the impact of computer software plug-in technology and further research and application.

First of all, the plug-in technology from the computer software to understand the principle is to grasp the following two core concepts: the first is the dynamic link library, it is a can be used simultaneously by multiple program code and data library, rather than the executable file[1]. Dynamic link to the computer software process can obtain the original does not belong to the executable code for a function, so as to transfer the functions of the software system. There are dynamic and static modes in the retrieval mode. There are some differences between the two methods of retrieval for different computer software systems. Computer software to take what 
function to retrieve the way, depending on the plug-in software system, but its ultimate goal is to enhance the efficiency of the system, making the system more convenient to operate. The second is the interface, which is the entity itself will provide an abstraction to the outside world, used for internal operations to fly away from the external communication method [2]. The role of the interface is to achieve the translation of language and entities, is a transitional way, it is not directly into the system of communication language, but need to be translated through the interface, which is bound to have some additional burden. The interface has a hardware interface and the software interface for the computer, no doubt computer software system plug-in technology is a software interface, is related to the subsidiary functions into the system through the interface.

Second, from the type of computer software plug-ins point of view, there are three main categories:

The first is a simple plug-in, which is similar to the command, is widely used in computer software system plug-in type. However, the low degree of freedom of this plug-in, is the smaller text, there are many shortcomings. It cannot be freely to transfer, and the mutual compatibility of the system have a higher degree of dependence. In other words, this plug-in often cannot be grafted in different systems. However, it also has strengths or advantages, which is relatively simple to do plug-ins, convenient, time-consuming less, many non-professional computer personnel or programmers can also complete the simple plug-in related production and use. In the process of use, this kind of plug-in is more simple, the user need only according to the set procedures, according to a defined procedure for operating the user input in advance.

The second is the language plug-in. This kind of plug-in needs to be applied to a language script, which is more complex than a simple plug-in that is similar to a command. In the plug-in production process, the development of software using some special language script to explain the connotation, which can access to the program. Due to the use of a scripting language, such plug-in sentence cannot need other tools to make plug-ins, the software itself can be achieved, thus combine plug-in and system together, has a very good use of some software of the computer in the office automation.

The third is the existing program environment plug-ins. The free degree of this kind of plug-in is relatively high, the software developer can fully display their own creative thinking, for the actual needs of software production, integration of many functions, in order to achieve higher performance software system program. The program system has set up the interface independently, in order to guarantee the environmental plug-in unit can visit the program resource freely. This plug-ins go beyond the general meaning of the plug-in, and to be an important part of the system, which greatly improving the performance of software systems. For the production of such plug-ins also become more complex, and plug-in interface requires a complex process of coordination, which is a professional programmer to complete, rather than the general people can complete.

In addition to the above classification, we can also based on other criteria for this analysis. The above classification is only from the complexity of the degree, from a simple plug-in to the language plug-in, and then to the environment plug-ins, plug-ins gradually increased, and the coordination between the performance is gradually improved. Of course, all kinds of plug-ins have their own advantages and disadvantages. The advantages and disadvantages of plug-in needs to be based on the applicable system. Different computer system software and performance requirements, determines the plug-in production and design.

\section{Research on the Function and Application of Computer Software Plug - in Technology}

There are many aspects of the computer software plug-in technology, which is the guarantee of the performance of the software. Broadly, its functions include: First, the computer software plug-in technology, can greatly enhance and expand the effectiveness of computer software, but also do not need to change the original computer software program. When the computer software interface is open, people can design the related plug-in according to the actual need, which makes the individual operation characteristic of the software more obvious. Different computer users may have the different requirements of computer system performance, which can compensate by 
software. Second, the computer software plug-in technology has very wide range of applications[3]. Such as e-commerce on the server needs, based on plug-in technology, you can adjust the performance of computer software for related applications to provide a more smooth configuration services, and these improvements will greatly enhance the computer in the production and life of people in many areas, which greatly enhance the efficiency of computer software. Based on these features, the computer software plug-in technology has been widely used, become one of the security of the computer function. The use of plug-in technology, as well as optimal combination of different functions of the combination to enhance the efficiency of the computer. Of course, in this process, the software plug-in in use need to constantly update, to better enhance the advantages of computer plug-ins to meet different customer needs. The application of this kind of plug-in technology, which slows down the pressure of the computer software technology update, reduces the cycle of software technology development and related cost investment.

With the wide use of computer and the development of computer software technology, the application of computer software plug-in technology is gradually becoming the focus by people. These application research not only focus on the development of new plug-in technology, but also how to optimize the existing plug-in technology to extend the effectiveness of plug-in technology. From the perspective of applied research, there are the following major aspects:

The first is from the overall point of view from the hair, combined with the needs of the reality of the computer function, the integration of the existing plug-in module optimization, will be integrated into all the original split module. In this way, it is possible to better characterize individual elements, to ensure that they not only function as a whole, but also to ensure that the individual modules can work together. At present, the module of the computer software plug-in technology is more serious, thus affecting the overall effectiveness of the plug-in play, limiting the use of computer software plug-in technology applications.

The second is from vertical development to horizontal development. Previously, the use of plug-in technology more emphasis on the longitudinal development of different software systems, to the depth of the development and application of functions. This is no doubt indispensable. However, for computer software, horizontal development is also extremely important. This is related to the different software system plug-ins to enhance the coordination and compatibility between them, and puts a higher demand on the cross-border research of program developers.

The third is the need to further strengthen the training of technical personnel. Applied research comes from the needs of real life, but it is also professional. The demand for talent is great. Therefore, in order to meet the needs of this development, we need to attach importance to computer professional training. To strengthen the theoretical knowledge of programmer time and ability, to ensure that they can make their own to get maximum play, develop the more practical, more efficient computer plug-in and the software system [4]. The use of plug-in technology in the development of computer software, can make the computer software become more systematic and professional.

The fourth is the need to further combine the actual needs of reality, to strengthen the application of plug-in technology. In the process of system development, to a certain extent, taking into account the needs of the application plug-in, so as to set aside the possibility of the application of plug-in technology. According to the needs of social and economic development, for the different needs of the production and living sector, to strengthen the use of plug-in technology, to better meet the actual needs of real life. It is very necessary to enhance the application of computer software plug-in technology research.

\section{References}

[1] Guo Zhisong. Analysis of the influence of plug-in technology on computer software [J]. electronic technology and software engineering, 2014, 2:118.

[2] Jiang Chuan. Plug-in technology and application in computer software [J]. Digital technology and application, 2013, 1:94. 
[3] Chang Jiang. Application of plug-in technology in computer software [J]. electronic technology and software engineering, 2013, 11:39.

[4] Zhong Zhen. Plug in technology in the application of computer software technology [J]. electronic production, 2015, 6:70. 